\title{
Immunopathology of Terminal Complement Activation and Complement C5 Blockade Creating a Prosurvival and Organ-protective Phenotype in Trauma
}

\author{
Zhangsheng Yang ${ }^{1}$, Miles Nunn ${ }^{2}$, Tuan Le ${ }^{1}$, Milomir Simovic $^{1}$, Peter Edsall ${ }^{1}$, Bin Liu ${ }^{1}$, \\ Johnny Barr ${ }^{1}$, Brian Lund ${ }^{3}$, Crystal Hill-Pryor ${ }^{4}$, Anthony Pusateri ${ }^{5}$, Leopoldo Cancio ${ }^{1}$, \\ and Yansong $\mathrm{Li}^{1}$
}

${ }^{1}$ US Army Institute of Surgical Research

${ }^{2}$ Akari Therapeutics Plc, London, United Kingdom

${ }^{3} 59$ th Medical Wing

${ }^{4}$ US Army Medical Research and Development Command

${ }^{5}$ Naval Medical Research Unit San Antonio

February 7, 2022

\begin{abstract}
Background and Purpose: Traumatic hemorrhage (TH) is the leading cause of potentially preventable deaths that occur during the prehospital phase of care. No effective pharmacological therapeutics are available for critical TH patients yet. Here, we identify terminal complement activation (TCA) as a therapeutic target in combat casualties and evaluate the efficacy of TCA inhibitor (nomacopan) on organ damage and survival in vivo. Experimental Approach: Complement activation products and cytokines were analyzed in plasma from 54 combat casualties, and the correlations between activated complement pathway(s) and the clinical outcomes in trauma patients were assessed. Nomacopan was administrated to rats subjected to lethal TH (blast injury and hemorrhagic shock). Effects of nomacopan on $\mathrm{TH}$ were determined using survival rate, organ damage, physiologic parameters, and laboratory profiles. Key Results: Early TCA was found to be associated with systemic inflammatory responses and clinical outcomes in this trauma cohort. Lethal TH in the untreated rats induced early TCA that correlated with severity of tissue damage and mortality. The addition of nomacopan to a damage control resuscitation (DCR) protocol significantly inhibited TCA, decreased local and systemic inflammatory responses, improved hemodynamics and metabolism, attenuated tissue and organ damage, and increased survival. Conclusion and Implications: Our findings reveal that early TCA represents a rational therapeutic target for trauma patients; and nomacopan as a prosurvival and organ-protective drug, could emerge as a promising adjunct to DCR that may significantly reduce the morbidity and mortality in severe TH patients while awaiting transport to critical care facilities.
\end{abstract}

\section{Hosted file}

Complement C5 inhibition for trauma and hemorrhagic shock.pdf available at https://authorea. com/users/459274/articles/555589-immunopathology-of-terminal-complement-activation-andcomplement-c5-blockade-creating-a-prosurvival-and-organ-protective-phenotype-in-trauma 